\title{
HOAG'S OBJECT, REMNANT OF A VANISHED BAR?
}

\author{
Tarsh Freeman ${ }^{1}$, Sethanne Howard ${ }^{2}$, Gene G. Byrd ${ }^{3}$ \\ ${ }^{1}$ Bevill State Community College \\ ${ }^{2}$ USNO, Retired \\ ${ }^{3}$ University of Alabama
}

\begin{abstract}
This beautiful object in the sky, named after its discoverer, Art Hoag, is an interesting galaxy, little studied. A study of what could form that unusual ring structure is the goal of this paper.
\end{abstract}

Hoag's Object is named after its discoverer, Art Hoag (1950), an astronomer of the $20^{\text {th }}$ century who, in 1950 , thought it to be a peculiar galaxy in the constellation of Serpens. Today it is considered an atypical $16^{\text {th }}$ magnitude ring galaxy. A nearly perfect ring of young hot blue stars circle the older round yellow nucleus. The ring and the nucleus have about the same luminosity. The galaxy is about 600 million light-years away. Given that distance we can compute the size of the galaxy. Let kly stand for 1000 light years; kpc stand for 1000 parsecs; Mpc stand for one million parsecs, " stand for arcsecond, and assume a Hubble constant $=70$ $\mathrm{km} / \mathrm{s} / \mathrm{Mpc}$. The diameter of the 6 " inner core of the galaxy is about $17 \pm$ $0.7 \mathrm{kly}(6 \mathrm{kpc})$ while the surrounding ring has an inner $28^{\prime \prime}$ diameter of 75 $\pm 3 \mathrm{kly}(24.8 \pm 1.1 \mathrm{kpc})$ and an outer $45^{\prime \prime}$ diameter of $121 \pm 4 \mathrm{kly}(39.9 \pm$ $1.7 \mathrm{kpc}$ ). This makes it slightly larger than the Milky Way (O'Connell et al 1974). The ring rotates with $V_{\max }=300(+100,-60) \mathrm{km} \mathrm{s}^{-1}$ and is inclined about $19^{\circ} \pm 5^{\circ}$ to the plane of the sky (Schweizer et al 1987). This gives the inner part of the ring a rotation period about 254 million years. Schweizer also determined the HI mass of the ring to be $1.8 \times 10^{10} M_{\odot}$, where $M_{\odot}$ stands for solar masses. The ring shows knotty structure and gaseous emission lines indicative of young stars. As rare as this type of galaxy is, oddly, another more distant ring galaxy can be seen, between the nucleus and the outer ring at the 0100 (one o'clock) position. 


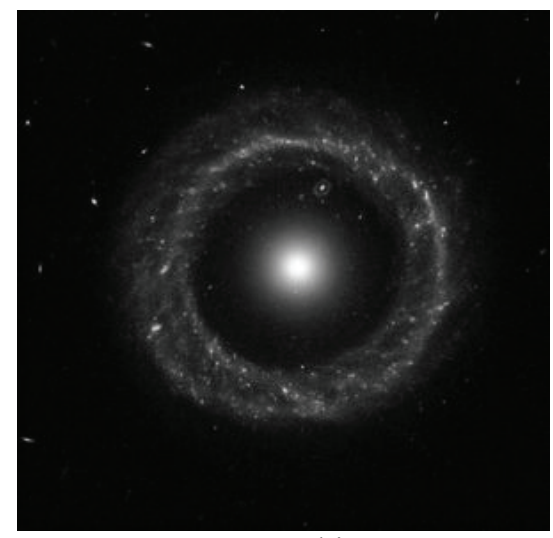

Hoag's Object

Courtesy NASA/ESA

Many of the details of the galaxy remain a mystery, foremost of which is how it formed. "Classic" ring galaxies are generally formed by the collision of a small galaxy with a larger spiral galaxy. This collision produces a cascade in the disk which leads to a characteristic ring-like appearance. However, there is no sign of any nearby second galaxy that would have acted as the "bullet", and the core of Hoag's Object has a very low velocity relative to the ring, making the typical formation hypothesis unlikely. Even though there is no sign of a second galaxy, Byrd and Howard (1992) showed that interactions between galaxies are frequent and most large galaxies are surrounded by very small companion galaxies. Therefore, the "bullet" may be too small to see. Even so, tidal interactions with small companions usually leave behind tidal tails or spiral structure.

It has been suggested that Hoag's Object might be a product of an extreme "bar instability" which occurred a few billion years ago in a barred spiral galaxy. Schweizer, et al., (1987) claim that this is an unlikely hypothesis because the nucleus of the object appears to be a normal spheroid, whereas the nucleus of a barred spiral galaxy is bar shaped or elongated. However, they admit evidence is somewhat thin for this particular dispute to be settled satisfactorily. Interestingly, a few galaxies share the primary characteristics of Hoag's Object, including a bright detached ring of stars, but their centers are elongated or barred, and they may exhibit some spiral structure. While none match Hoag's Object in symmetry, this handful of galaxies are known as Hoag-type galaxies.

According to Friedli and Pfenniger (1991) galaxy dissipative processes can transform an otherwise steady bar accreting mass into the center into a shorter, faster, and weaker triaxial ellipsoid, similar to the 
bulges of normal spirals. They show an example where after 8 Gyr dissipation brought $1.3 \%$ of the total mass inside $0.2 \mathrm{kpc}$, increasing the local average density by a factor of 14 . When seen face-on in this example the resulting bulge resembles a spheroid. A rapid inflow of gas clouds in systems with bars and a central mass concentration can occur. As the central mass grows, one or two unavoidable low order gravitational resonances develop which cause the main elongated plane orbit family supporting the bar to be replaced by planar orbit families perpendicular to the bar which can then no longer be sustained. The dissipation laws (Pfenniger and Norman, 1990) include dynamical friction, friction laws proportional to $v^{\mathrm{n}}, \mathrm{n}>1$, and more complicated laws depending on the projection of the velocity vector parallel and/or perpendicular to the effective potential.

It has also been suggested (Schweizer, 1987) that a major "accretion event", in other words a collision or a capturing of one galaxy by another, caused the formation of the galaxy we see today. The event likely took place at least 2-3 billion years in the past, and may have resembled the processes that form polar-ring galaxies.

We decided to try a computer simulation to see if we could produce the observed ring structure with a bar which grows then disappears. Can the ring which is thus created survive for a long time after the bar is gone? Astronomers build a galaxy out of individual points, plop the points in a computer - the bigger the better, and then they let the points gravitationally interact and follow the patterns that develop over time. The gravitational law used is the standard Newtonian formula:

$$
F_{i j}=G \frac{m_{i} m_{j}}{r_{i j}^{2}}
$$

where the force $F_{i j}$ between particles $i$ and $j$ is given by their masses and the inverse square of the distance between them. $G$ is the gravitational constant.

To simulate the galaxy we use a sophisticated $n$-body code first written by Miller (1968, 1970, 1974, 1978a, 1978b). The code has been through many tests and additions over time and, despite its age, retains its usefulness. The code has an exponential two dimensional polar grid on which it solves Poisson's equation for each time step,

$$
\nabla^{2} \Phi(\vec{r})=-4 \pi \rho(\vec{r})
$$

where $\Phi$ is the gravitational potential and $\rho$ is the density at point $r$. 
The coordinates of each grid point in the grid are $(\phi, \alpha)$ where $\alpha$ (the radial coordinate) runs from 1 to 26 , and $\phi$ (the azimuthal coordinate) runs from 1 to 36. An inert spherical halo potential can be added to the simulation. Star and gas particles partially fill the grid at the beginning of the simulation according to the Mestel (1963) potential. The Mestel potential, $\Phi(r)$, for a star of mass $m$ orbiting in a disk of radius $R$ is:

$$
\Phi(r)=\left\{\begin{array}{c}
m v_{0}^{2} \ln (r / 2 R) \\
\frac{-2 m v_{0}^{2}}{\pi} \sum_{\ell=0}^{\infty} \frac{1 \cdot 3 \cdot 5 \cdot \ldots(2 \ell+1)}{2^{\ell} \ell !(2 \ell+1)^{3}}\left(\frac{2 R}{r}\right)^{2 \ell+1}
\end{array}\right.
$$

where the top value is for $r<R$ and the bottom is for $r>R$. The quantity $v_{0}$ is the constant rotation velocity. The Mestel potential produces a flat rotation curve where $\Omega=V / r, \Omega$ is the angular orbital velocity, $V$ is the orbital velocity, $r$ is the radial distance from the center. The velocity, $V$, is approximately constant over the disk. A Mestel disk is an observationally good approximation to a thin galaxy disk. The inert halo potential at each grid point is $\operatorname{VLC}(\phi, \alpha)$ and remains the same throughout the simulation. The crossing time for the simulations is 50 time steps; i.e., it takes 50 times steps for a particle to move a distance of one disk radius. When a particle reaches a radius less than 1 radial unit it is set back to 1 and returned to the simulation; i.e., particles entering the center cross through and stay "in play".

We ran several simulations with a companion tidally triggering the disk and could not reproduce the observed structure. Either tidal tails result or the ring that appears is small, of low luminosity, leaving most of the particles in the remaining visible disk.

We then adjusted the code to include a slowly rotating bar. There is no companion galaxy. The $n$ disk bodies again start out in equilibrium, satisfying the conservation laws statistically and following a Mestel potential. To ensure a quiet start in equilibrium the small difference between the initial analytic disk and halo potential and numerical disk potential, $\operatorname{VLCTEM}(\phi, \alpha)$ is included in the halo potential to maintain disk numerical stability. This difference is very small but significant. The disk potential is adjusted each time step, ISTEP, according to the positions and velocities of the particles in the disk. The total number of time steps in the simulation is STEPS. 
The bar potential starts out small and builds over time. The bar potential is added to the constant halo potential each time step which then feeds into the solution for Poisson's equation across the whole disk. There are three degrees of freedom for the bar: the amplitude, $A M P 1$, of the bar potential, its turning rate, $T U R$, and the scaling for angle. The amplitude of the bar potential, $A M P$, follows a sinusoidal pattern:

$$
A M P=(\sin (I S T E P / 3628) \cdot A M P 1
$$

where $A M P 1$ and $T U R$ are then chosen to match the observed galaxy profile. $A M P 1$ (best value $=0.25$ ) sets the amplitude of the sinusoidal bar pattern, and the best value for the angle scaling is 3628. TUR (best value = 3.552) sets the turning rate of the bar. Therefore the amplitude is a slowly varying sinusoidal function that increases as the simulation proceeds and then falls off. After it reaches a peak the amplitude falls off and completely dissipates by time step 3628 . The amplitude, $A M P$, is added to the halo potential as a sinusoidal series as follows:

$\operatorname{VHALO}(\phi, \alpha)=\operatorname{VLC}(\phi, \alpha)+\operatorname{VLCTEM}(\phi, \alpha)+$

$A M P \times((L C(\phi, \alpha)+V C T E M(\phi, \alpha)) \times \cos ((2 \cdot 2 \pi \cdot \phi / 36)+(T U R \cdot($ ISTEP $) / S T E P S))+$

$A M P \times((L C(\phi, \alpha)+V C T E M(\phi, \alpha)) \times \cos ((4 \cdot 2 \pi \cdot \phi / 36)+(T U R \cdot($ ISTEP $) / S T E P S))+$ $A M P \times((V C(\phi, \alpha)+V L C T E M(\phi, \alpha)) \times \cos ((6 \cdot 2 \pi \cdot \phi / 36)+(T U R \cdot($ ISTEP $) / S T E P S))$.

The figure shows the result of a 7,000 time step simulation with 60,000 particles, all gas, in the disk. The ring has formed, the bar has gone, and many particles cluster around the core.

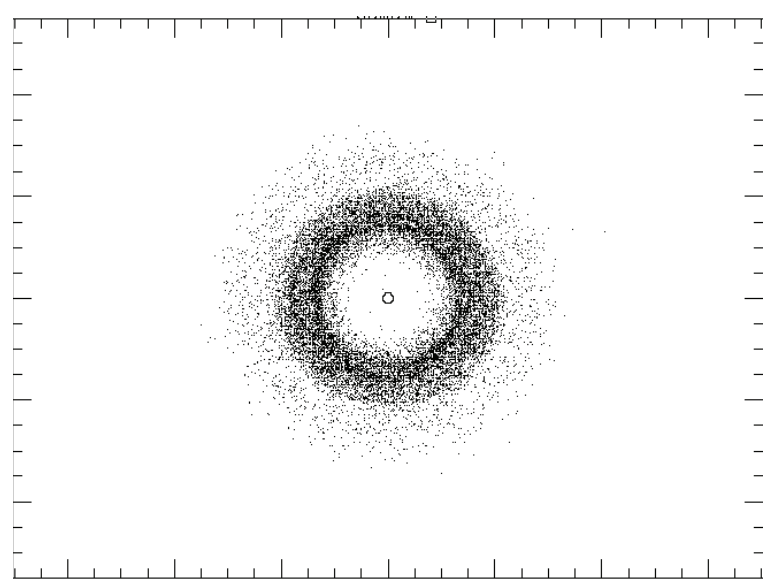

The simulated Hoag's Object on the left, $n=60,000 ; 7,000$ steps into the simulation. Note how there are more particles on the inner part of the ring similar to Hoag's Object. 
The bar has dissipated by this time. Many particles have gathered near the center where they bounce back and forth across $r=0$, as if they were spiraling into a massive black hole or gathering into a spheroid. They stay near the center and do not enter the void. There are more particles in the center than there are in the ring. There are very few particles left in the void (only 9).

It takes 50 time steps for a particle to travel one disk radius or about 1.6 million years per time step. At 7,000 time steps we are $1.1 \times 10^{10}$ years into the simulation - about 43 ring rotations. The simulation uses a very heavy halo that is 15 times the mass in the disk. A light halo (mass less than 5 times the mass in the disk) does not clear out the void.

The following diagram shows the percentage of particles gathered into the center and gathered into the ring, with a mere 9 in the gap between the two. About $62 \%$ of the total particles are in the center. About $38 \%$ of the total particles are in the ring. The number of particles in center gives a mass, therefore, of approximately 36,000 particles, and the ring has a mass of approximately 23,000 particles. Since the HI mass of the ring is about $1.8 \times 10^{10} M_{\odot}$, this means that the galaxy center may have at least a mass of $2.9 \times 10^{10}{ }_{M_{\odot}}$.

PARTICLE COUNT

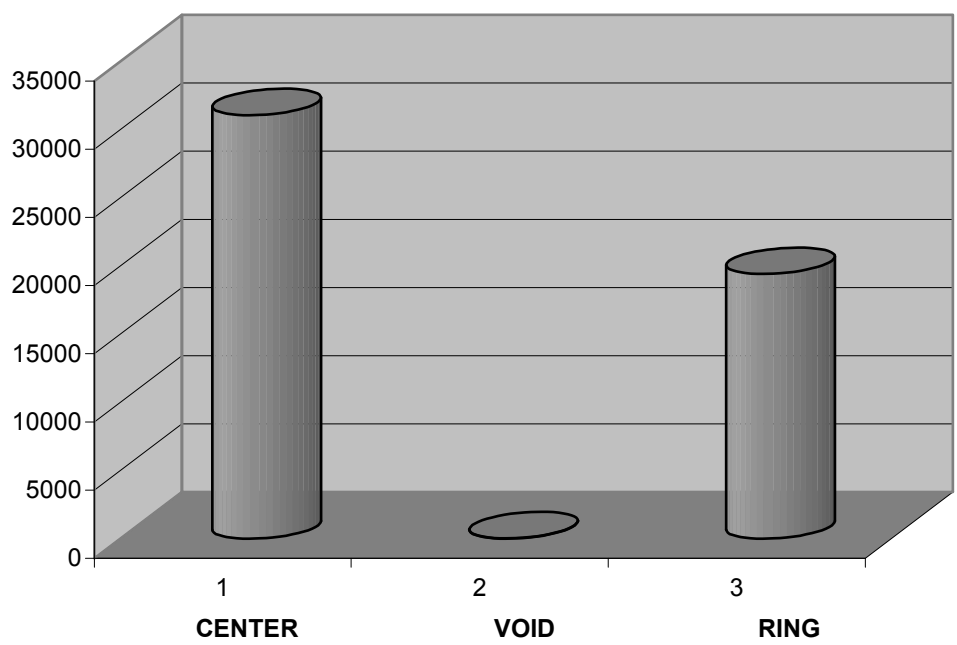

The figure shows how empty the void is. More particles gather in the center than in the ring. 
The bar dissipates by time step 3628 so at time step 7000 the ring has survived at least twice as long as the bar has. This is in agreement with the idea that the ring formed from a bar instability that occurred some few billion years ago. Although we show that many particles gather in the center we cannot with this simulation estimate the shape of the center. So we cannot address Schweizer's objection to the bar instability hypothesis.

\section{References}

1950, Hoag, Arthur A. Astronomical Journal, 55, 170

1963, L. Mestel, Astrophysical Journal, 126, 553

1968, R. H. Miller, Astrophysical Journal, 151,699

1970, R. H. Miller, Astrophysical Journal, 161, 90

1974, R. H. Miller, Astrophysical Journal, 190, 539

1974, O'Connell, Robert W.; Scargle, Jeffrey D.; Sargent, W. L. W., Astrophysical Journal, 191, 61

1978a, R. H. Miller, Astrophysical Journal, 223, 811

1978b, R. H. Miller, Astrophysical Journal, 224, 32

1987, Schweizer, F., Ford, W. Kent, Jr.; Jederzejewski, R.; Giovanelli, R., Astrophysical Journal, 320, 454

1990, D. Pfenniger and C. Norman, Astrophysical Journal, 363, 391

1991, D. Friedli and D. Pfenniger, Dynamics of Galaxies and their Molecular Cloud Distribution, IAU Symposium, 146, 362

1992, G. Byrd, S. Howard, Astronomical Journal, 103, 1089 
The world according to Hubble Is a giant expanding bubble.

Far away things have fled

With a shift to the red

As if we were the cause of the trouble.

Paul Arveson

Fellow, WAS

Your loverly limerick on Hubble

Will save us a great deal of trouble.

Our readers hate blanks,

So we send you our thanks,

And use it we will--on the double !

Vary Coates 